\title{
Digital Navigation Services
}

David J. Rosen, Newsome Associates

This issue's Technology Solutions column does not follow the typical order of education challenge followed by technology solution, although ultimately one of the main purposes of a digital navigator solution is to address an education challenge, especially when remote or online learning is involved, and when in-person learning options are unavailable. The challenge in this column is: good computer or Chromebook access to broadband internet from home for education purposes. The solution, this time, focuses on the human help needed to address the challenges of reliable broadband internet access from home and help with the digital literacy skills needed for online learning.

Technology Challenges: Helping adult learners obtain affordable home broadband access to the internet; affordable digital devices such as discounted desktop and laptop computers or Chromebooks for use at home; and digital literacy skills needed for learning online and possibly also for work or finding employment, finding healthrelated information and services, or to meet other pressing needs for navigating digital devices and the internet.

Human Solution: Digital navigation services provided by a digital navigator

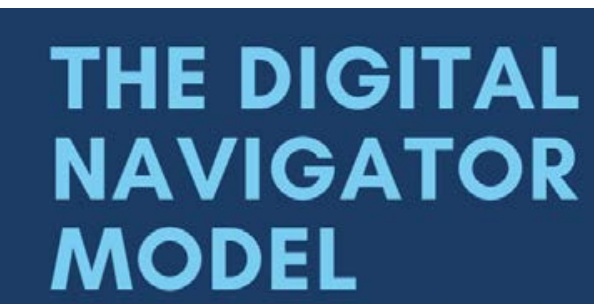

"Adding Digital Equity to Our Social Safety Net",

The parts of an Infographic appearing in this article were published in a report Literacy Minnesota produced with funding from the Minnesota Department of Education. They are included here with Literacy Minnesota's permission. The whole infographic will be found at https://www. literacymn.org/sites/default/files/uploads/MDE docs/ Digital Navigator Model Infographic.png and also at https:// www.digitalinclusion.org/wp-content/uploads/dlm_ uploads/2021/04/DN-Infographic.pdf

\section{What Is a Digital Navigator?}

Someone who helps people to find their way to and in the online world, for example, who helps them:

- Find affordable internet access from home or elsewhere

- Find an affordable internet-accessible digital device such as a desktop or laptop computer, electronic tablet, or Chromebook

- Acquire the digital skills they need to accomplish online learning, online health tasks, online job 
application or job-related goals, to access online banking and complete online banking tasks, and for other important needs that can be met online

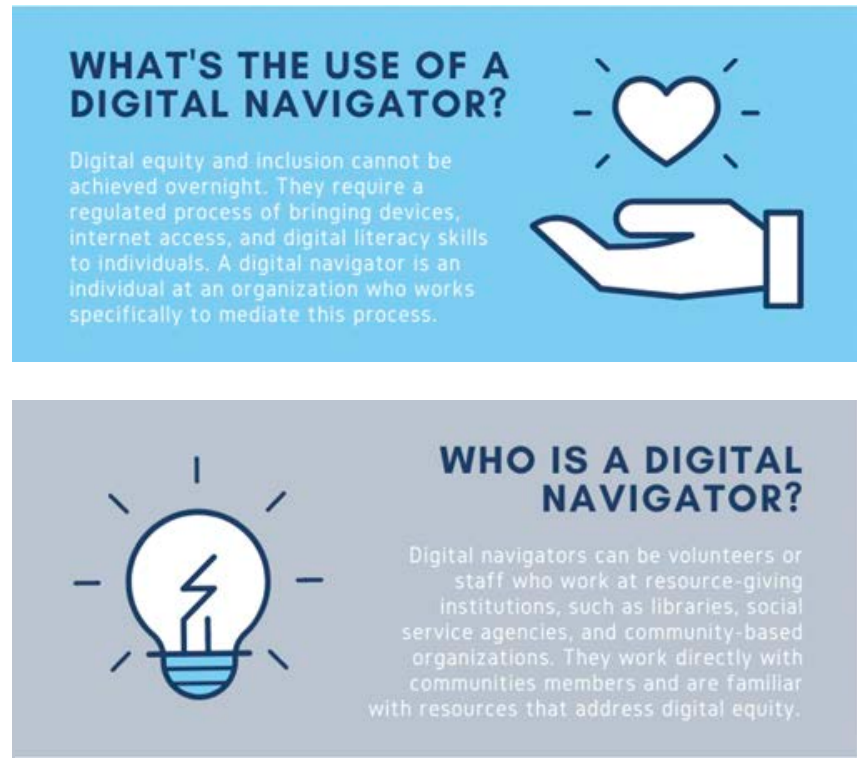

Where Can Local Digital Navigators Be Found?

Digital navigators can work for or volunteer with:

- Public libraries

- Adult basic skills education programs and schools

- Mayor's offices

- Hospitals and community health centers

- Companies and corporations

- Labor unions

- Universities and colleges

- Other organizations; or coalitions, collaboratives, or partnerships of organizations

Currently they can be found in 18 states.

A digital navigator could be a new full-time or parttime in-person, on-phone, and/or online position, or a set of digital navigation services integrated into an existing job, for example, in the job of a reference librarian, community health worker, or a company's human resources department staff member.

\section{Why Is This Role Needed and What Kinds of Digital Navigation Services Are Provided?}

The COVID-19 pandemic has dramatically demonstrated that students in $\mathrm{K}-12$, higher education and adult basic skills education need reliable and affordable home access to the internet, and that they may also need a home digital device and/or specific digital literacy skills. Digital navigators, available by phone, in person, and for those who have reliable broadband access by internet, can help them, one-on-one, and sometimes in small groups, to meet these needs for technology and digital literacy skills. Sometimes digital navigators address a wide range of needs; at other times they may address a small number of contextualized needs. For example, a digital health navigator may focus on how to help patients use:

- A hospital health portal, for example to schedule appointments, get test results, and communicate with their health provider team members

- Telehealth or telemedicine services and/or

- A digital device such as a smartphone that is capable of transmitting vital data to their health provider team in connection with a particular disease.

\section{Digital Navigators in Adult Basic Skills Education Programs}

In addition to helping students access the internet or get an affordable home digital device, a digital navigator in an adult basic skills education program may focus on helping students get the digital skills they need to use the program or school's online learning platform. This may be its course management system or learning management system, or a teacher-created class website that includes assignments and links to text-, audio- and video-based learning resources. 
In at least one adult basic skills program, in Boston, "trained bilingual Digital Navigators attend trainings to help students remotely onboard to using apps through a variety of ways, such as one-on-one sessions after class or during class in a breakout room. Navigators assist students with day-to-day digital inclusion problems and also coach them on how to use the programs more proficiently to learn. Navigators are evolving to become classroom assistants, having an even more direct role in the students' learning" (Sharma et al., 2021, para. 5).

In some cases-Digital Charlotte's digital navigator program in North Carolina is one example"digital navigators have adapted into the role of guide-supporting clients through each steprather than merely sending them off with referrals without ensuring all their digital inclusion needs are met" (Sharma et al., 2021, para. 9).

\section{How Is Help With Digital Navigation Skills Provided?}

Through as-needed, in-person, one-on-one and small group support when possible, and/or by voice telephone, email, texting, and video chat.

\section{How Do Those Who Need These Services Find Out About Them?}

- From community organizations as diverse as a fire station, a cultural heritage center, a community health center or hospital, or a faith-based organization

- Through social media

- From adult basic skills program practitioners and from statewide organizations

- From laptop lending programs, for example at libraries or in school districts or community colleges

\section{How Can I Learn More About Digital Navigators and Digital Navigation Services?}

- The Digital US Digital Navigators web pages https://digitalus.org/digital-navigators/

- The Digital US Digital Navigator Resources hub https://digitalus.org/digital-navigatorresources/

- $\quad$ The Digital US Digital Navigator Playbook https://digitalus.org/digital-navigatorplaybook/ A comprehensive guide that details steps and considerations as programs and systems set up or improve their Digital Navigator services

- The Digital US Blog Series: https://edtech. worlded.org/digital-navigators-awarenessof-services-impact/ ; https://edtech.worlded. org/digital-navigators-adaptability-key-indesign/ and https://edtech.worlded.org/digitalnavigators-trust-drives-impact/

- The National Digital Inclusion Alliance Digital Navigator Model https://www.digitalinclusion. org/digital-navigator-model/

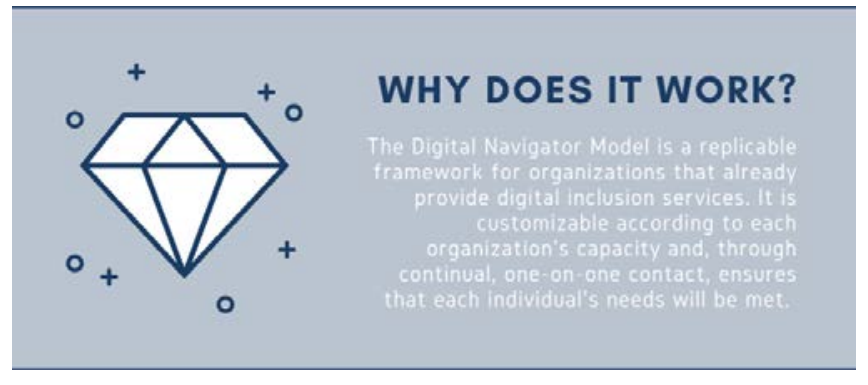

HOW DOES IT WORK?

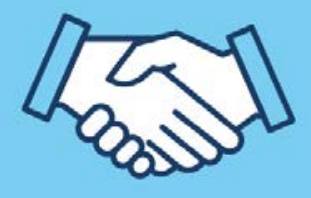




\section{Reflections}

Because of the pandemic, a great many people including legislators and other policy makers and private funders are now aware of the extensive digital divide in the United States and how it has directly affected the learning goals of children and adults. There are a number of federal, state, and local public and private sources of funding now that adult basic skills programs may be able to access to address this problem, and because of advocacy organizations like the National Digital
Inclusion Alliance, more funders now understand that in addition to internet access and home computers teaching digital literacy skills is also essential for many students. There are many ways to address this problem, of course, including digital skills courses, integrating digital skills into basic skills or occupational courses, and through technology coaches. Digital navigation services support is a new, and promising role that many adult basic skills programs, or the collaboratives of which they are a part, might consider.

\section{References}

Sharma, P., Gonzalez, C., Cabrera Holguin, N., \& Nuchprayoon, P. (2021, May 28). Digital navigators: Adaptability key in design. EdTech Center. https://edtech.worlded.org/digital-navigators-adaptability-key-in-design/ 